Taras Shevchenko National University of Kyiv,

Faculty of Radiophysics, Electronics, and Computer Systems

(4g, Prosp. Academician Glushkov, Kyiv 03680, Ukraine; e-mail: volodumur88@volia.ua)

\title{
HYBRID RESONANCE IN THE SPLIT-RING RESONATOR/FERRITE STRUCTURE IN THE C-BAND
}

\begin{abstract}
The resonance properties of a structure consisting of a split-ring resonator (SRR) and a ferrite have been studied experimentally. The obtained hybrid resonance is shown to include several peaks, which are located in a vicinity of the fundamental SRR resonance. The resonance frequency is found to depend on the magnetic field with a frequency tuning constant of $450 \mathrm{kHz} / \mathrm{Oe}$.

Ke ywords: split-ring resonator, metamaterial, ferrite, C-band, frequency tuning.
\end{abstract}

\section{Introduction}

Split-ring resonators (SRRs) are artificial structures, in which the relative magnetic susceptibility $\mu$ can become negative under certain conditions [1]. SRRs are used for the creation of artificial media with the negative $\mu$ [2] (the so-called metamaterials) and as elements in various filters $[3,4]$ and aerials $[5,6]$. Their popularity can be explained by the relative simplicity of their construction and the presence of materials that are used for their fabrication. Plenty of constructive solutions have been proposed for them $[7,8]$. However, both the simplest SRR variant, this is a split metallic ring, and more complicated variants possess a substantial shortcoming: they are not frequency-tunable. Therefore, researchers have proposed several variants in order to solve this problem. All of them are reduced to a combination of the SRR with additional parts that allow the equivalent capacity of the structure to be changed; in particular, these are a varactor [9], liquid crystals [10], lightly doped silicon [11], and others. Usually such structures are considerably bigger than an ordinary SRR.

(C) V.S. CHORNYI, S.L. SKRIPKA, B.YA. LENYK, I.V. BASIUK, O.Y. NECHYPORUK, 2016

ISSN 2071-0194. Ukr. J. Phys. 2016. Vol. 61, No. 8
Not so long ago, there was proposed a structure that allows the frequency characteristics of the resonator to be controlled by changing its equivalent inductance. The result was reached in the experimental work [12] by combining an SRR and a ferrite. The model for this artificial medium was developed in work [13]. The authors of the cited works showed that the fundamental resonance (as well as the higher-order resonances [13]) becomes split into two peaks. Depending on the position of the ferromagnetic resonance (FMR), either the left or the right peak prevails. However, this undoubtedly interesting and promising idea has not been developed further. It should also be noted that the researchers confined themselves to only one type of SRR and ferrite (the yttrium iron garnet).

On the other hand, ferromagnets are widely used to create metamaterials. In particular, from a variety of works on this subject, we would like to mention the following ones, in which strontium-doped lanthanum manganite was used. In work [14], a double negative medium was created by combining this ferromagnet with a photonic crystal. In work [15], it was used to create a periodic structure described by the Drude formula for the electron gas (i.e. a negative value of $\epsilon$ was obtained). At the same time, by varying the 


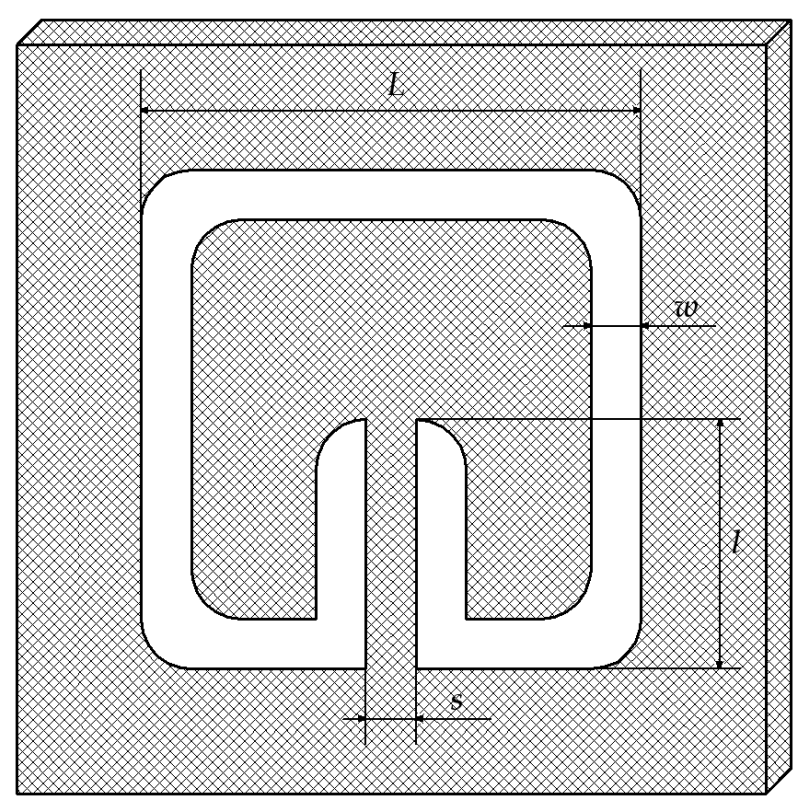

Fig. 1. SRR with the rectangular geometry. Metallization is designated by white

external dc magnetic field, the FMR was obtained in the same frequency range, which made it possible to obtain a negative beam refraction. In work [16], the influence of the impurity concentration on properties of lanthanum manganite as a metamaterial with controllable frequency characteristics was studied.

Ferrites themselves are known to be a material, in which the negative value of $\mu$ can be obtained and, simultaneously, whose frequency characteristic can be varied by means of an external magnetic field. However, ferrites are more complicated to manufacture and more expensive materials than SRRs. It is worth noting that a combination of those materials makes it possible to obtain the frequency tuning in an interval located near the fundamental SRR resonance, whereas the FMR can occur even several octaves lower or higher. In other words, to obtain a set of materials with controllable parameters over several frequency ranges, one needs not possess a collection of ferrites. It would be enough to fabricate structures with SRRs and use them with one ferrite material only. Moreover, this combined structure does not require that its components should be rigidly fastened with one another. We should also note that this structure is more compact than its analogs with the variable equivalent capacitance.
This work was aimed at experimentally researching the hybrid resonance in a structure created from a SRR and a ferrite, and the possibility of its frequency tuning by an external magnetic field. In accordance with the IEEE Std. 521-2002 and IEEE Standard Letter Designations for Radar-Frequency Bands, the researches were carried out in the frequency C-band extending from 4 to $8 \mathrm{GHz}$.

\section{Description of the Structure and the Experimental Installation}

Split-ring resonators were proposed for the first time in work [1]. They consist of metallic rings deposited onto a dielectric substrate. There are two types of SRR excitation: magnetic and electric. When the SRR is excited by an external ac magnetic field directed normally to the ring plane, a current runs from one ring to another through the cuts between them and, as a result, acquires the form of a strong bias current. Some SRR types can be excited by an electric field oriented in the plane of ring cuts [7]. In this case, the motion of charges gives rise to the formation of an electric dipole. For resonators with the inverse symmetry, the electric excitation is impossible $[7,17]$. The case where the SRR has excitations of two types is called the cross-polarization effect. It can be regarded as negative, because the corresponding amplitude-frequency characteristic of the resonator depends on the position of the latter with respect to the electric field. At the same time, this effect can be used to control the amplitude-frequency characteristic, although within a narrow interval (in comparison with the frequency-tunable resonators).

Generally speaking, there are a lot of SRR variants $[7,18]$. We selected an SRR with a rectangular geometry (Fig. 1) [7]. The SRR of this type revealed itself well, while creating an artificial medium, in which an object can be hidden from the external probing radiation [19]. This type of SRRs is characterized by the cross-polarization effect. The researches were carried out by magnetically exciting the structure.

Six resonators were arranged on every specimen. The resonator characteristics were determined by the electromagnetic simulation with the use of the software package HFSS (ANSYS, Inc). For the resonance frequency $f_{0}=5 \mathrm{GHz}$, the following parameters of the structure were obtained: the length $L=6 \mathrm{~mm}$, the length $l=2.8 \mathrm{~mm}$, the width $s=0.8 \mathrm{~mm}$,

ISSN 2071-0194. Ukr. J. Phys. 2016. Vol. 61, No. 8 
the width $w=0.4 \mathrm{~mm}$, and the structure period amounted to $8 \mathrm{~mm}$. As the dielectric substrate, we used RT/duroid 5880 (for more details, see work [20]) with the following parameters: $\epsilon=2.2 \pm 0.02$ (at $10 \mathrm{GHz}$ ), a dielectric loss tangent of 0.0009 (at $10 \mathrm{GHz}$ ), and a thickness of $125 \mu \mathrm{m}$. The metallization layer thickness was $17 \mu \mathrm{m}$, and the specimen dimensions amounted to $48 \times 8 \mathrm{~mm}^{2}$.

Two types of ferrites were used. Their main parameters are quoted in Table 1 . The ferrites were put on the resonator top. The magnetization was transverse. The FMR frequency did not exceed $2 \mathrm{GHz}$.

In order to observe the fundamental SRR resonance, it is necessary to provide a required distribution of the electromagnetic field. In the case of magnetic excitation, we have to provide an ac magnetic field with the strength vector directed perpendicularly to the SRR surface. To satisfy this condition, we used a rectangular waveguide with a crosssection of $48 \times 24 \mathrm{~mm}^{2}$. The external magnetic field was created with the help of an electromagnet. Its strength reached 640 Oe. As a microwave source and for the visualization of resonance characteristics of the structure, we used a vector network analyzer Rohde\&Schwarz ${ }^{\circledR}$ ZVA8. The interval of working frequencies for this analyzer extended from $300 \mathrm{kHz}$ to $8 \mathrm{GHz}$.

\section{Measurement Results and Their Discussion}

In Fig. 2, the frequency dependences of the amplitude and phase of the transmission coefficient for the fundamental SRR resonance are depicted. It should be noted that here we have a number of contributions to the transmission coefficient. Similar phenomena were also observed, e.g., in work [21].

The best results were obtained for the structure SRR/40SCh2. Figure 3 demonstrates the corresponding resonance characteristics obtained at external magnetic field strengths of 70,360 , and 640 Oe. The resonance curve had four peaks. At a field strength of $70 \mathrm{Oe}$, they had the following values of resonance frequency and absorption: $6.288 \mathrm{GHz}(-11.96 \mathrm{~dB})$, $6.383 \mathrm{GHz}(-14.2 \mathrm{~dB}), 6.713 \mathrm{GHz}(-19.25 \mathrm{~dB})$, and $6.935 \mathrm{GHz}(-11.81 \mathrm{~dB})$. The first peak remained almost invariant, as the magnetic field increases: $6.298 \mathrm{GHz}(-11.63 \mathrm{~dB})$ at 640 Oe. The fourth peak went beyond the measurement limits in this case (at approximately 420 Oe), but before this
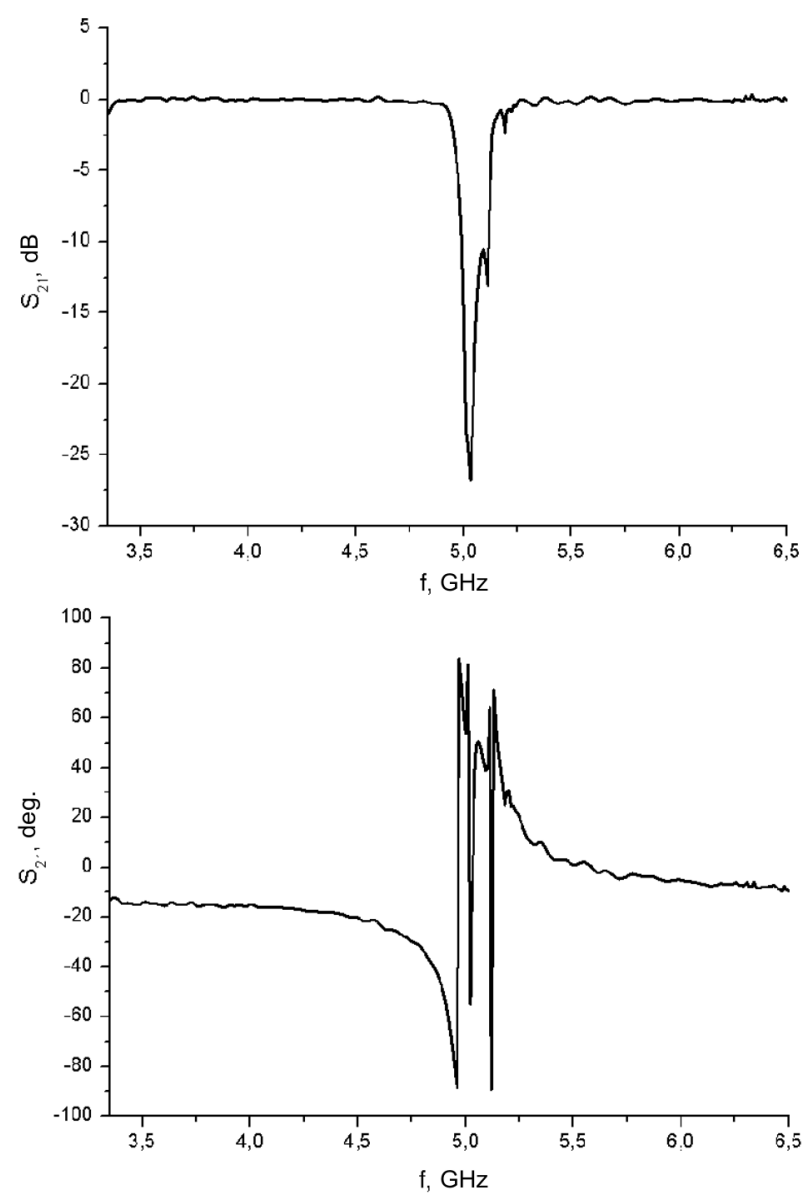

Fig. 2. Amplitude and phase of the transmission coefficient for the fundamental SRR resonance

event, it shifted with a frequency tuning constant of $166.3 \mathrm{kHz} / \mathrm{Oe}$.

The parameters of the second and third peaks are quoted in Table 2. The rate of resonance frequency variation amounted to $286 \mathrm{kHz} / \mathrm{Oe}$ for the second peak and to $239 \mathrm{kHz} / \mathrm{Oe}$ for the third one. It should also be noted that the resonance band width increased with the magnetic field. In addition, the amplitude of the second peak became almost twice as large.

Table 1. Main parameters

of the ferrites used in experiment

\begin{tabular}{|c|c|c|c|c|c|}
\hline $\begin{array}{c}\text { Ferrite } \\
\text { grade }\end{array}$ & Size, $\mathrm{mm}^{3}$ & $\begin{array}{c}4 \pi M_{S} \pm 5 \%, \\
\text { Gs }\end{array}$ & $\begin{array}{c}\epsilon^{\prime} \pm \\
\pm 5 \%\end{array}$ & $\begin{array}{c}\operatorname{tg} \delta_{\epsilon} \times \\
\times 10^{4}\end{array}$ & $\begin{array}{c}\Delta H, \\
\text { Oe }\end{array}$ \\
\hline 10SCh6 & $48 \times 9 \times 1$ & 1780 & 15.1 & 2 & 35 \\
40 SCh2 & $54 \times 12.5 \times 1$ & 800 & 14.2 & 2 & 45 \\
\hline
\end{tabular}




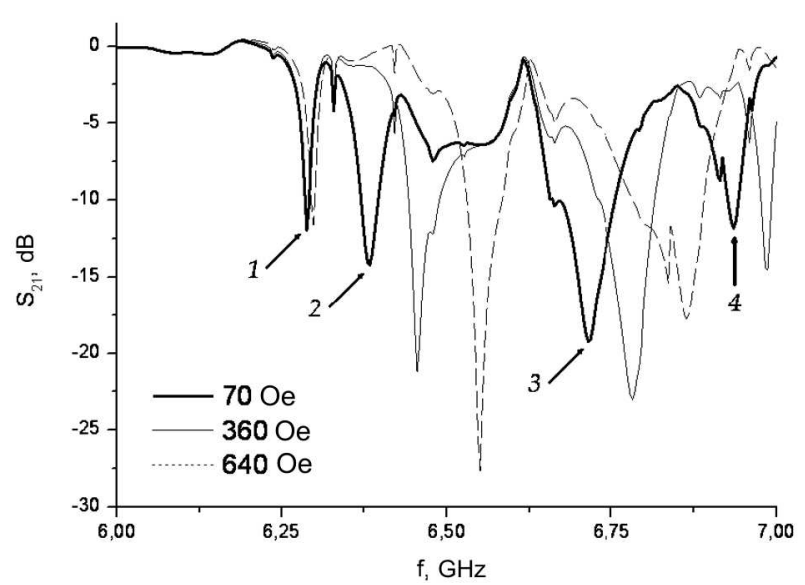

Fig. 3. Frequency dependence of the transmission coefficient for the structure SRR/40SCh2

Table 2. Parameters of the second and third peaks in the resonance curve for the structure SRR/40SCh2

\begin{tabular}{|c|c|c|c|c|}
\hline$H, \mathrm{Oe}$ & Peak & $f_{0}, \mathrm{GHz}$ & $\Delta f(-3 \mathrm{~dB}), \mathrm{MHz}$ & $L, \mathrm{~dB}$ \\
\hline \multirow[t]{2}{*}{70} & 2 & 6.383 & 44 & -14.2 \\
\hline & 3 & 6.713 & 145 & -19.25 \\
\hline \multirow[t]{2}{*}{125} & 2 & 6.4 & 41 & -18.77 \\
\hline & 3 & 6.737 & 160 & -20.37 \\
\hline \multirow[t]{2}{*}{185} & 2 & 6.41 & 34 & -19.69 \\
\hline & 3 & 6.744 & 170 & -21.53 \\
\hline \multirow[t]{2}{*}{300} & 2 & 6.441 & 198 & -19.68 \\
\hline & 3 & 6.768 & 186 & -22.86 \\
\hline \multirow[t]{2}{*}{360} & 2 & 6.455 & 177 & -21.2 \\
\hline & 3 & 6.782 & 190 & -23.02 \\
\hline \multirow[t]{2}{*}{475} & 2 & 6.492 & 143 & -25.53 \\
\hline & 3 & 6.812 & 230 & -21.78 \\
\hline \multirow[t]{2}{*}{535} & 2 & 6.509 & 126 & -25.84 \\
\hline & 3 & 6.826 & 255 & -20.95 \\
\hline \multirow[t]{2}{*}{590} & 2 & 6.53 & 120 & -26.84 \\
\hline & 3 & 6.833 & 274 & -18.41 \\
\hline \multirow[t]{2}{*}{640} & 2 & 6.55 & 113 & -27.63 \\
\hline & 3 & 6.863 & 281 & -17.76 \\
\hline
\end{tabular}

When using 10SCh6, the resonance characteristic had one strongly pronounced peak (Fig. 4). There were also two small peaks (at 3.5 and $5.2 \mathrm{GHz}$ ), which shifted as the magnetic field changed, but their amplitudes did not exceed $-3 \mathrm{~dB}$. Therefore, we decided

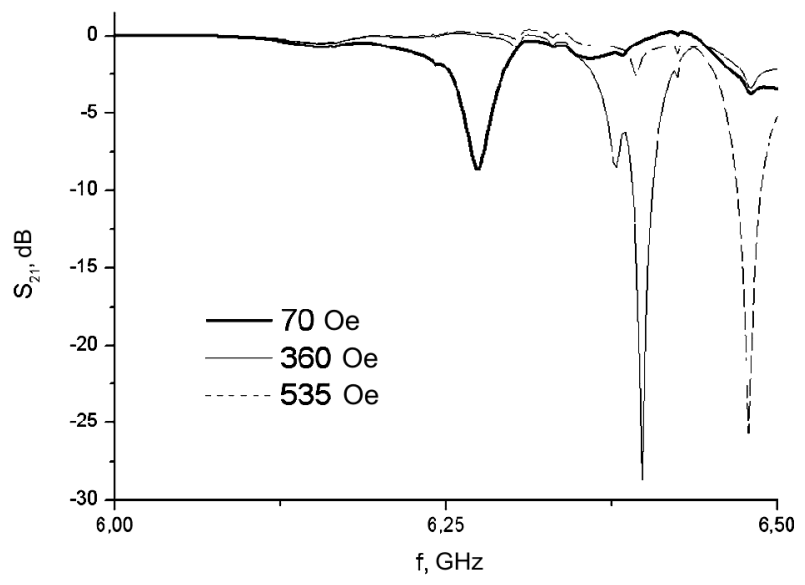

Fig. 4. Frequency dependence of the transmission coefficient for the structure SRR/10SCh6

Table 3. Resonance curve parameters for the structure SRR/10SCh6

\begin{tabular}{|r|l|c|c|}
\hline$H, \mathrm{Oe}$ & $f_{0}, \mathrm{GHz}$ & $\Delta f(-3 \mathrm{~dB}), \mathrm{MHz}$ & $L, \mathrm{~dB}$ \\
\hline 70 & 6.274 & 32 & -14.2 \\
125 & 6.281 & 35 & -18.77 \\
185 & 6.32 & 35 & -19.69 \\
245 & 6.341 & 27 & -19.68 \\
300 & 6.363 & 24 & -21.2 \\
360 & 6.398 & 35 & -25.53 \\
420 & 6.419 & 33 & -25.84 \\
475 & 6.448 & 33 & -26.84 \\
535 & 6.478 & 44 & -27.63 \\
\hline
\end{tabular}

to concentrate our attention on the largest among the obtained peaks. Its parameters are listed in Table 3. The amplitude of this peak increased more than by a factor of three. The corresponding rate of resonance frequency variation amounted to $450 \mathrm{kHz} / \mathrm{Oe}$.

It is worth noting that different numbers of peaks were obtained for different ferrites. This issue concerns the interaction of SRR with ferrite when the latter is in the domain region. The corresponding problem is not trivial and should be considered separately. The contribution is also given by higher-order resonances, but, unfortunately, their research goes beyond the capability limits of our experimental installation.

\section{Conclusions}

In this work, we presented the experimental results obtained, while measuring the frequency response of

ISSN 2071-0194. Ukr. J. Phys. 2016. Vol. 61, No. 8 
structures consisting of an SRR and a ferrite. The SRRs with the rectangular geometry and two different ferrites were used. It is shown that the obtained resonance characteristic includes several peaks. This fact can be explained by supposing that the fundamental resonance only looks like a single narrow peak. In fact, it consists of several peaks that are practically superimposed. While calculating the SRR parameters, the method of equivalent resonance circuit was used, when the resonator was substituted by an LC contour. Actually, ring resonators consist of several contours (especially their more complicated variants). However, in this case, the corresponding specification gives rise to more complicated calculations without providing the appreciable improvement of their accuracy (at least at frequencies of several gigahertz). Therefore, when SRRs are used in practice without ferrites, this feature is not taken into consideration, as a rule. The application of ferrites gives rise to the increase of the distance between the peaks.

We have also demonstrated that the obtained resonance curve, shifts if an external magnetic field is applied. The corresponding frequency tuning constant amounted to $450 \mathrm{kHz} / \mathrm{Oe}$ for the $10 \mathrm{SCh} 6$ ferrite and to $286 \mathrm{kHz} / \mathrm{Oe}$ for the $40 \mathrm{SCh} 2$ one.

1. J.B. Pendry, Phys. Rev. Lett. 85, 3966 (2000).

2. R.A. Shelby, D.R. Smith, and S. Schultz, Science 292, 77 (2001).

3. I. Gil, F. Martín, X. Rottenberg, and W. De Raedt, Electron. Lett. 43, 1153 (2007).

4. K.C. Yoon, J.H. Kim, and J.C. Lee, Microw. Opt. Techn. Lett. 53, 2174 (2011).

5. J. Kim, C.S. Cho, and J.W. Lee, Electron. Lett. 42, 315 (2006).

6. H. Nornikman, O.T. Kean, A.B. Hisham, A.A.M.Z. Abidin, S.W. Yik, and O.M. Azlishah, Australian J. Basic Appl. Sci. 8, 262 (2014).

7. R. Marqués, F. Martín, and M. Sorola, Metamaterials with Negative Parameters. Theory, Design, and Microwave Applications (Wiley-Interscience, New York, 2007).

8. E. Ekmekci and G. Turhan-Sayan, Prog. Electromag. Res. 12, 35 (2009).
9. I. Gil, J. Bonache, J. García-García, and F. Martín, IEEE Trans. Microwave Theory Tech. 54, 2665 (2006).

10. Q. Zhao, L. Kang, B. Du, B. Li, and J. Zhou, Appl. Phys. Lett. 90, 011112 (2007).

11. A. Degiron, J.J. Mock, and D.R. Smith, Opt. Express 15, 1115 (2007).

12. L. Kang, Q. Zhao, H. Zhao, and J. Zhou, Opt. Express 16, 12 (2008).

13. J.N. Gollub, J.Y. Chin, T.J. Cui, and D.R. Smith, Opt. Express 17, 4 (2009).

14. M.K. Khodzitsky, T.V. Kalmykova, S.I. Tarapov, D.P. Belozorov, A.M. Pogorily, A.I. Tovstolytkin, A.G. Belous, and S.A. Solopan, Appl. Phys. Lett. 95, 082903 (2009).

15. M.K. Khodzitsky, S.I. Tarapov, D.P. Belozorov, A.M. Pogorily, A.I. Tovstolytkin, A.G. Belous, and S.A. Solopan, Appl. Phys. Lett. 97, 131912 (2010).

16. D.P. Belozorov, A.A. Girich, S.I. Tarapov, A.M. Pogorily, A.I. Tovstolytkin, A.G. Belous, and S.A. Solopan, AIP Adv. 4, 037116 (2014).

17. R. Marqués, F. Medina, and R. Rafii-El-Idrissi, Phys. Rev. B 65, 144440 (2002).

18. L. Solymar and E. Shamonina, Waves in Metamaterials (Oxford Univ. Press, Oxford, 2009).

19. D. Schurig, J.J. Mock, B.J. Justice, S.A. Cummer, J.B. Pendry, A.F. Starr, and D.R. Smith, Science 214, 977 (2006).

20. https://www.rogerscorp.com/documents/606/acm/RTduroid-5870-5880-Data-Sheet.pdf.

21. J.D. Baena, R. Marqués, J. Martel, and F. Medina, in Proceedings of the IEEE-AP/S International Symposium on Antennas and Propagation, Columbus (2003), p. 106.

Received 18.11.15.

Translated from Ukrainian by O.I. Voitenko

B.С. Чорний, С.Л. Скрипка,

Б.Я. Леник, І.В. Басюк, О.Ю. Нечипорук

ГІБРИДНИЙ РЕЗОНАНС

В СТРУКТУРІ ПОДВІЙНИЙ КІЛЬЦЕВИЙ

РЕЗОНАТОР/ФЕРИТ В С-ДІАПАЗОНІ

$\mathrm{P}$ е $з$ ю м е

У даній роботі представлено експериментальні результати дослідження резонансних властивостей структури, яка складається $з$ подвійних кільцевих резонаторів (ПКР) та фериту. Показано, що отриманий гібридний резонанс складається $з$ декількох піків та знаходиться в околі основного резонансу ПКР. Отриманий резонанс керується магнітним полем. Частотна перебудова становила 450 кГц/Е. 